\title{
Stochastic Simulation of Biochemical Systems: In Memory of Dan T. Gillespie's contributions
}

\author{
Yang $\mathrm{Cao}^{1} \cdot$ Petzold Linda $^{2} \cdot$ Effrosyni Seitaridou $^{3}$
}

Published online: 1 July 2019

(c) Society for Mathematical Biology 2019

On April 19, 2017, sad news hit all of us in the research communities of computational biology, stochastic simulation and applied physics. Our beloved friend, Dan T. Gillespie, a research physicist best known for the stochastic simulation algorithm (SSA, also known as the Gillespie Algorithm), passed away at his home in Castaic California at the age of 78 .

The impact of Dan Gillespie's work on the field of stochastic physics, computational biology and other related areas has been huge. When he first presented his original work in Gillespie (1976), it was called "completely wrong" by some prominent researchers in the field of chemistry. Certainly, it was far ahead of its time. In the late $1990 \mathrm{~s}$, it came to light that intrinsic stochasticity, arising from extremely small populations of key molecular species, was playing an important role in cell biology. The Gillespie algorithm was perfectly suited for simulating these systems. Since that time, it has been widely used in cell biology. Dan Gillespie's foundational publications would eventually receive over ten thousand citations, and he was well recognized as one of the founders of the field of stochastic physics in biology. His research contributions spanned a wide range of fields, including cloud physics, random variable theory, Brownian motion, Markov process theory, electrical noise, light scattering in aerosols, and quantum mechanics.

The contributions in this special issue come from researchers working in many different disciplines that have benefitted from Dan Gillespie's pioneering work. The Gillespie algorithm (Gillespie 1976, 1977) is an important modeling and simulation method, but it also suffers from inefficiency when applied to large scale systems. He and his collaborators developed different methods to improve the algorithm, such as the tau-leaping method (Gillespie 2001; Rathinam et al. 2003; Cao et al. 2005b), and the

\footnotetext{
$\bowtie \quad$ Yang Cao

ycao@cs.vt.edu

1 Department of Computer Science, Virginia Tech, Blacksburg, VA 24061, USA

2 Department of Computer Science and Mechanical Engineering, University of California at Santa Barbara, Santa Barbara, CA 93106, USA

3 Department of Physics, Oxford College of Emory University, 100 Hamill St., Oxford, GA 30054, USA
} 
slow-scale SSA method (Cao et al. 2005a, c). This special issue includes a review article on rejection-based exact methods (Thanh 2018), improvements to the tau-leaping method (Beentjes and Baker 2018) and the S-leaping method (Lipková et al. 2018), and numerical analysis of the hybrid ODE/SSA method (Chen et al. 2018). In addition to the development of simulation algorithms, there have been efforts to improve the efficiency of the many simulations that are required to obtain accurate approximations to the probability density functions of the chemical species. Simulation strategies and numerical algorithms have been proposed to enable the use of fewer simulations while still maintaining high accuracy. This special issue includes work on the multilevel approach (Engblom 2018), the variance-reduced simulation method (Maginnis et al. 2019) for general Markov chains, and the low variance coupling method (Anderson and Yuan 2018).

The original Gillespie algorithm is physically accurate only for systems that are both dilute and well-mixed in the reactant (solute) molecules. An extension of the SSA for systems that are not well-mixed is the reaction-diffusion SSA (RD-SSA). It divides the system volume into subvolumes or "voxels", which are small enough that each can be considered to be well-mixed. Chemical reactions are then considered to occur inside individual voxels and are modeled using the SSA, while diffusion is modeled via jumps from a subvolume to one of its neighbors. In this way, the Gillespie algorithm has been extended to the challenging field of spatial stochastic modeling. This special issue features several contributions in this area: Grima gives a review for spatial stochastic modeling (Smith and Grima 2018), Kang and Erban (2019) present an analysis for multiscale reaction-diffusion system and Markov Chain, and Lötstedt (2018) presents a linear noise approximation for spatially dependent biochemical networks.

To highlight Dan Gillespie's impact on related fields, this special issue also features contributions that are related to his work from other areas, such as the network-free simulation method (Suderman et al. 2018), rare event analysis (Roh 2018), sensitivity analysis for multiscale stochastic systems (Gupta and Khammash 2018), and an application of stochastic dynamics to simulation of eukaryotic flagellar growth (Rathinam and Sverchkov 2018).

These articles are by no means, or even close to a full list of work that benefited from Dan Gillespie's great scientific career. We hope that they serve as milestones in memory of a brave pioneer, a close friend, a devoted mentor, and a great physicist.

\section{References}

Anderson DF, Yuan C (2018) Low variance couplings for stochastic models of intracellular processes with time-dependent rate functions. Bull Math Biol. https://doi.org/10.1007/s11538-018-0430-6

Beentjes C, Baker R (2018) Quasi-Monte Carlo methods applied to tau-leaping in stochastic biological systems. Bull Math Biol. https://doi.org/10.1007/s11538-018-0442-2

Cao Y, Gillespie DT, Petzold L (2005a) The slow-scale stochastic simulation algorithm. J Chem Phys 122:014116

Cao Y, Gillespie DT, Petzold L (2005b) Avoiding negative populations in explicit tau leaping. J Chem Phys 123:054104

Cao Y, Gillespie DT, Petzold L (2005c) Multiscale stochastic simulation algorithm with stochastic partial equilibrium assumption for chemically reacting systems. J Comput Phys 206:395-411 
Chen M, Wang S, Cao Y (2018) Accuracy analysis of hybrid stochastic simulation algorithm on linear chain reaction systems. Bull Math Biol. https://doi.org/10.1007/s11538-018-0461-z

Engblom S (2018) Stochastic simulation of pattern formation in growing tissue: a multilevel approach. Bull Math Biol. https://doi.org/10.1007/s11538-018-0454-y

Gillespie DT (1976) A general method for numerically simulating the stochastic time evolution of coupled chemical reactions. J Comput Phys 22(4):403-434

Gillespie DT (1977) Exact stochastic simulation of coupled chemical reactions. J Phys Chem 81(25):23402361

Gillespie DT (2001) Approximate accelerated stochastic simulation of chemically reacting systems. J Chem Phys 115:1716

Gupta A, Khammash M (2018) Sensitivity analysis for multiscale stochastic reaction networks using hybrid approximations. Bull Math Biol. https://doi.org/10.1007/s11538-018-0521-4

Kang HW, Erban R (2019) Multiscale stochastic reaction-diffusion algorithms combining Markov chain models with stochastic partial differential equations. Bull Math Biol. https://doi.org/10.1007/s11538019-00613-0

Lipková J, Arampatzis G, Chatelain P, Menze B, Koumoutsakos P (2018) S-leaping: an adaptive, accelerated stochastic simulation algorithm, bridging $\tau$-leaping and R-leaping. Bull Math Biol. https://doi.org/10. 1007/s11538-018-0464-9

Lötstedt P (2018) The linear noise approximation for spatially dependent biochemical networks. Bull Math Biol. https://doi.org/10.1007/s11538-018-0428-0

Maginnis PA, West M, Dullerud GE (2019) Exact variance-reduced simulation of lattice continuoustime Markov chains with applications in reaction networks. Bull Math Biol. https://doi.org/10.1007/ s11538-019-00576-2

Rathinam M, Sverchkov Y (2018) Stochastic dynamics of eukaryotic flagellar growth. Bull Math Biol. https://doi.org/10.1007/s11538-018-0427-1

Rathinam M, Petzold L, Cao Y, Gillespie DT (2003) Stiffness in stochastic chemically reacting systems: the implicit tau-leaping method. J Chem Phys 119:12784-94

Roh MK (2018) Data-driven method for efficient characterization of rare event probabilities in biochemical systems. Bull Math Biol. https://doi.org/10.1007/s11538-018-0509-0

Smith S, Grima R (2018) Spatial stochastic intracellular kinetics: a review of modelling approaches. Bull Math Biol. https://doi.org/10.1007/s11538-018-0443-1

Suderman R, Mitra ED, Lin YT, Erickson KE, Feng S, Hlavacek WS (2018) Generalizing Gillespie's direct method to enable network-free simulations. Bull Math Biol. https://doi.org/10.1007/s11538018-0418-2

Thanh VH (2018) A critical comparison of rejection-based algorithms for simulation of large biochemical reaction networks. Bull Math Biol. https://doi.org/10.1007/s11538-018-0462-y

Publisher's Note Springer Nature remains neutral with regard to jurisdictional claims in published maps and institutional affiliations. 